\title{
ANALYSIS OF FLEXURAL VIBRATION OF VISCOELASTICALLY DAMPED SANDWICH PLATES
}

\author{
J.-F. $\mathrm{HE}$ \\ Department of Engineering Mechanics, Tsinghua University, Beijing, The People's Republic of China \\ AND \\ B.-A. MA \\ Institute of Mechanics, Academia Sinica, Beijing, The People's Republic of China
}

(Received 20 May 1987, and in revised form 2 February 1988)

\begin{abstract}
The simplified governing equations and corresponding boundary conditions of flexural vibration of viscoelastically damped unsymmetrical sandwich plates are given. The asymptotic solution to the equations is then discussed. If only the first terms of the asymptotic solution of all variables are taken as an approximate solution, the result is identical with that obtained from the Modal Strain Energy (MSE) Method. As more terms of the asymptotic solution are taken, the successive calculations show improved accuracy. With the natural frequencies and the modal loss factors of a damped sandwich plate known, one can calculate the response of the plate to various loads providing a reliable basis for engineering design.
\end{abstract}

\section{INTRODUCTION}

It is known that structural vibration can be reduced by utilizing layers of viscoelastic damping material $[1,2]$. One effective approach is the constrained layer damping treatment. A constrained damped plate is generally an unsymmetrical sandwich plate, in which a layer of viscoelastic damping material is sandwiched between a primary structural layer and a constraining layer. The structural layer and the constraining layer may be made of different material. In general they are of unequal thicknesses. Such a sandwich plate will provide both strength and rigidity and yet have a low response to vibration over a wide frequency range.

The governing equations of flexural vibration of symmetrical sandwich plates have been given in reference [3]. Successively, a set of governing equations for the vibratory bending of unsymmetrical sandwich plates was given in references $[4,5]$. It is a set of twelfth-order partial differential equations including bending-extension coupling. Solving the equations is difficult. In recently published papers [6-8] the flexural vibrations of constrained damped plates with complex shapes and various boundary conditions have been computed by finite element methods, the mechanical plate model presented in reference [4] having been used. The viscoelastic core was modelled with solid elements and the face layers were modelled with plate elements. The number of degrees of freedom of all the elements was so large that the work was expensive. In reference [8] it was suggested that available design charts be used insofar as possible. However, the number of design charts is limited. Because of this, the analysis and design of unsymmetrical sandwich plates are restricted. It is the aim of this paper to present a set of simplified governing equations for unsymmetrical sandwich plates, not including bending-extension coupling, and to discuss its asymptotic solution in terms of real values. 


\section{THE GOVERNING EQUATIONS OF FLEXURAL VIBRATION}

To derive the governing equations of flexural vibration of unsymmetrical sandwich plates, the following assumptions are made: (1) the face-layers are elastic and isotropic and suffer no transverse shear deformation; (2) the core carries transverse shear, but no in-plane stresses; it is linearly viscoelastic and has a complex shear modulus; (3) no slip occurs at the interfaces of the core and face-layers; all points on a normal to the plate move with the same transverse displacement; (4) though the metallic material of the structural layer and the constraining layer may be different, the values of their Poisson ratios may be approximately equal; (5) when the sandwich plate is in flexural vibration, the in-plane inertia effects of the plate are ignored and only the transverse inertia effects are considered. Assumptions (1)-(3) are generally made. Both assumptions (4) and (5) are necessary to establish a set of simplified governing equations of unsymmetrical sandwich plates not including bending-extension coupling. The influence of the in-plane inertia forces upon dynamic characteristics of a plate was discussed in references $[4,5,9]$. In most cases assumption (5) is acceptable.

The unsymmetrical sandwich plate configuration is shown in Figure 1. The thicknesses of the constraining layer (face 1), the viscoelastic layer (face 2) and the structural layer (face 3 ) are $t_{1}, t_{2}$ and $t_{3}$ respectively. The transverse displacement of the plate in flexural vibration is $W(x, y, t)$. The in-plane displacements of the points in the middle planes of face 1 and face 3 are $U_{1}, V_{1}$ and $U_{3}, V_{3}$ respectively. The in-plane displacements of interfaces are $U_{12}, V_{12}$ and $U_{32}, V_{32}$ respectively. According to the assumptions, there are uniform transverse shear strain components $\gamma_{x z}$ and $\gamma_{y z}$ in the viscoelastic layer:

$$
\gamma_{x z}=\frac{U_{12}-U_{32}}{t_{2}}+\frac{\partial W}{\partial x}=\frac{U_{1}-U_{3}}{t_{2}}+\frac{c}{t_{2}} \frac{\partial W}{\partial x}, \quad \gamma_{y z}=\frac{V_{12}-V_{32}}{t_{2}}+\frac{\partial W}{\partial y}=\frac{V_{1}-V_{3}}{t_{2}}+\frac{c}{t_{2}} \frac{\partial W}{\partial y} .
$$

Here $c=t_{2}+\frac{1}{2}\left(t_{1}+t_{3}\right)$. The expression for the strain energy of the plate $U$ is

$$
U=\frac{1}{2} \iint\left\{\gamma_{1}\left[\left(\frac{\partial U_{1}}{\partial x}\right)^{2}+2 \nu \frac{\partial U_{1}}{\partial x} \frac{\partial V_{1}}{\partial y}+\left(\frac{\partial V_{1}}{\partial y}\right)^{2}+\frac{(1-v)}{2}\left(\frac{\partial U_{1}}{\partial y}+\frac{\partial V_{1}}{\partial x}\right)^{2}\right]\right.
$$
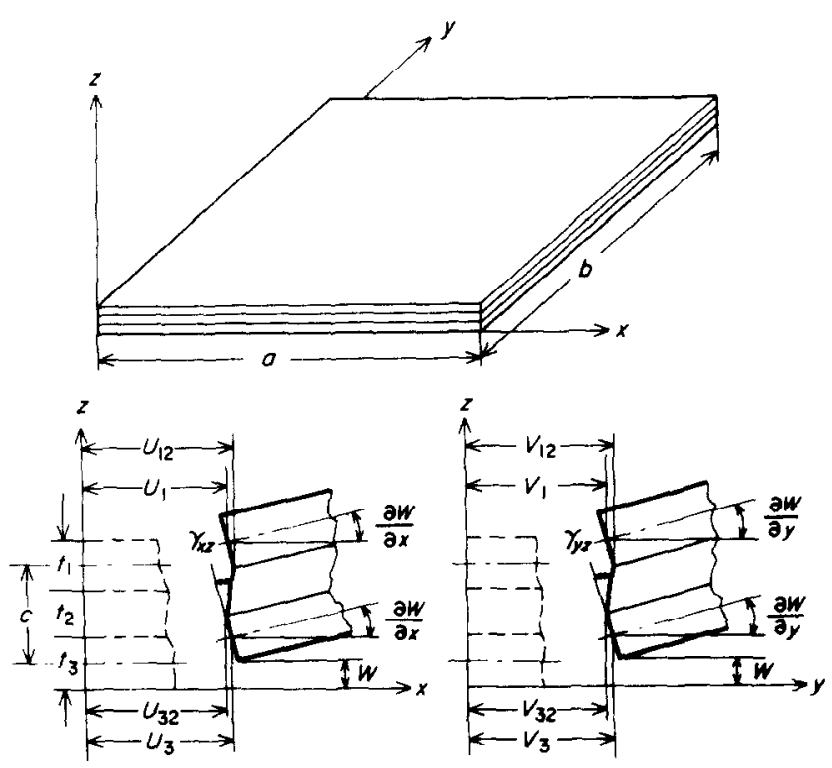

Figure 1. The dimensions and the displacements of a damped sandwich plate. 


$$
\begin{aligned}
& +\gamma_{3}\left[\left(\frac{\partial U_{3}}{\partial x}\right)^{2}+2 \nu \frac{\partial U_{3}}{\partial x} \frac{\partial V_{3}}{\partial y}+\left(\frac{\partial V_{3}}{\partial y}\right)^{2}+\frac{(1-\nu)}{2}\left(\frac{\partial U_{3}}{\partial y}+\frac{\partial V_{3}}{\partial x}\right)^{2}\right] \\
& +\left(D_{1}+D_{3}\right)\left[\left(\frac{\partial^{2} W}{\partial x^{2}}\right)^{2}+2 \nu \frac{\partial^{2} W}{\partial x^{2}} \frac{\partial^{2} W}{\partial y^{2}}+\left(\frac{\partial^{2} W}{\partial y^{2}}\right)^{2}+2(1-\nu)\left(\frac{\partial^{2} W}{\partial x \partial y}\right)^{2}\right] \\
& \left.+\frac{\gamma_{2}}{t_{2}^{2}}\left[\left(U_{1}-U_{3}+c \frac{\partial W}{\partial x}\right)^{2}+\left(V_{1}-V_{3}+c \frac{\partial W}{\partial y}\right)^{2}\right]\right\} \mathrm{d} x \mathrm{~d} y .
\end{aligned}
$$

Here

$$
\gamma_{1}=\frac{E_{1} t_{1}}{1-\nu^{2}}, \quad \gamma_{2}=G_{2} t_{2}, \quad \gamma_{3}=\frac{E_{3} t_{3}}{1-\nu^{2}}, \quad D_{1}=\frac{E_{1} t_{1}^{3}}{12\left(1-\nu^{2}\right)}, \quad D_{3}=\frac{E_{3} t_{3}^{3}}{12\left(1-\nu^{2}\right)},
$$

where $E_{1}$ and $E_{3}$ are the elastic moduli of the faces 1 and $3, \nu$ is their common Poisson ratio, $G_{2}$ is the shear modulus of the core 2 , which is taken as a real quantity temporarily. One can introduce

$$
\begin{gathered}
U_{m}(x, y, t)=\left[1 /\left(\gamma_{1}+\gamma_{3}\right)\right]\left[\gamma_{1} U_{1}(x, y, t)+\gamma_{3} U_{3}(x, y, t)\right], \\
V_{m}(x, y, t)=\left[1 /\left(\gamma_{1}+\gamma_{3}\right)\right]\left[\gamma_{1} V_{1}(x, y, t)+\gamma_{3} V_{3}(x, y, t)\right], \\
\psi_{x}(x, y, t)=(1 / c)\left[U_{1}(x, y, t)-U_{3}(x, y, t)\right], \quad \psi_{y}(x, y, t)=(1 / c)\left[V_{1}(x, y, t)-V_{3}(x, y, t)\right] .
\end{gathered}
$$

Here $U_{m}$ and $V_{m}$ may be regarded as the weighted mean in-plane displacements of the unsymmetrical sandwich plate, $\psi_{x}$ and $\psi_{y}$ are the rotary angles of a line connecting the two corresponding points at the middle planes of the faces 1 and 3 after bending. For the sake of convenience later, the following dimensionless variables are introduced:

$$
\begin{gathered}
\xi=x / a, \quad \eta=y / a, \quad u_{m}=U_{m} / a, \quad v_{m}=V_{m} / a, \quad w=W / a, \quad \psi_{\xi}=\psi_{x}, \\
\psi_{\eta}=\psi_{y}, \quad g=\gamma_{2}\left(\gamma_{1}+\gamma_{3}\right) a^{2} / \gamma_{1} \gamma_{3} t_{2}^{2}, \quad Y=\gamma_{1} \gamma_{3} c^{2} /\left(\gamma_{1}+\gamma_{3}\right)\left(D_{1}+D_{3}\right) .
\end{gathered}
$$

Here $a$ is a characteristic dimension of the plate, e.g., the length of an edge of a rectangular plate; $g$ and $Y$ are called the "shear parameter" and the "geometric parameter" respectively.

The strain energy $U$ can now be rewritten as

$$
\begin{aligned}
U= & \frac{1}{2}\left(\gamma_{1}+\gamma_{3}\right) a^{2} \iint\left[\left(\frac{\partial u_{m}}{\partial \xi}\right)^{2}+2 \nu \frac{\partial u_{m}}{\partial \xi} \frac{\partial v_{m}}{\partial \eta}+\left(\frac{\partial v_{m}}{\partial \eta}\right)^{2}+\frac{(1-\nu)}{2}\left(\frac{\partial u_{m}}{\partial \eta}+\frac{\partial v_{m}}{\partial \xi}\right)^{2}\right] \mathrm{d} \xi \mathrm{d} \eta \\
& +\frac{1}{2}\left(D_{1}+D_{3}\right) \iint\left\{\left[\left(\frac{\partial^{2} w}{\partial \xi^{2}}\right)^{2}+2 \nu \frac{\partial^{2} w}{\partial \xi^{2}} \frac{\partial^{2} w}{\partial \eta^{2}}+\left(\frac{\partial^{2} w}{\partial \eta^{2}}\right)^{2}+2(1-\nu)\left(\frac{\partial^{2} w}{\partial \xi \partial \eta}\right)^{2}\right]\right. \\
& +Y\left[\left(\frac{\partial \psi_{\xi}}{\partial \xi}\right)^{2}+2 \nu \frac{\partial \psi_{\xi}}{\partial \xi} \frac{\partial \psi_{\eta}}{\partial \eta}+\left(\frac{\partial \psi_{\eta}}{\partial \eta}\right)^{2}+\frac{(1-\nu)}{2}\left(\frac{\partial \psi_{\xi}}{\partial \eta}+\frac{\partial \psi_{\eta}}{\partial \xi}\right)^{2}\right] \\
& \left.+Y g\left[\left(\psi_{\xi}+\frac{\partial w}{\partial \xi}\right)^{2}+\left(\psi_{\eta}+\frac{\partial w}{\partial \eta}\right)^{2}\right]\right\} \mathrm{d} \xi \mathrm{d} \eta
\end{aligned}
$$

The kinetic energy of the plate $T$ is

$$
T=\frac{1}{2} \rho a^{4} \iint\left(\frac{\partial w}{\partial t}\right)^{2} \mathrm{~d} \xi \mathrm{d} \eta,
$$

where $\rho$ is the mass per unit area of the plate. 
According to Hamilton's principle, one can obtain a set of simplified governing equations of motion of unsymmetrical sandwich plates (in dimensionless form):

$$
\begin{gathered}
L_{1}\left(u_{m}, v_{m}\right) \equiv \frac{\partial^{2} u_{m}}{\partial \xi^{2}}+\frac{(1+\nu)}{2} \frac{\partial^{2} v_{m}}{\partial \xi \partial \eta}+\frac{(1-\nu)}{2} \frac{\partial^{2} u_{m}}{\partial \eta^{2}}=0, \\
L_{2}\left(u_{m}, v_{m}\right) \equiv \frac{\partial^{2} v_{m}}{\partial \eta^{2}}+\frac{(1+\nu)}{2} \frac{\partial^{2} u_{m}}{\partial \xi \partial \eta}+\frac{(1-\nu)}{2} \frac{\partial^{2} v_{m}}{\partial \xi^{2}}=0, \\
L_{3}\left(w, \psi_{\xi}, \psi_{\eta}\right) \equiv \frac{\partial^{2} \psi_{\xi}}{\partial \xi^{2}}+\frac{(1+\nu)}{2} \frac{\partial^{2} \psi_{\eta}}{\partial \xi \partial \eta}+\frac{(1-\nu)}{2} \frac{\partial^{2} \psi_{\xi}}{\partial \eta^{2}}-g\left(\psi_{\xi}+\frac{\partial w}{\partial \xi}\right)=0, \\
L_{4}\left(w, \psi_{\zeta}, \psi_{\eta}\right) \equiv \frac{\partial^{2} \psi_{\eta}}{\partial \eta^{2}}+\frac{(1+\nu)}{2} \frac{\partial^{2} \psi_{\xi}}{\partial \xi \partial \eta}+\frac{(1-\nu)}{2} \frac{\partial^{2} \psi_{\eta}}{\partial \xi^{2}}-g\left(\psi_{\eta}+\frac{\partial w}{\partial \eta}\right)=0, \\
L_{5}\left(w, \psi_{\xi}, \psi_{\eta}\right) \equiv \nabla^{4} w-Y g\left(\frac{\partial \psi_{\xi}}{\partial \xi}+\frac{\partial \psi_{\eta}}{\partial \eta}+\nabla^{2} w\right)+\frac{\rho a^{4}}{\left(D_{1}+D_{3}\right)} \frac{\partial^{2} w}{\partial t^{2}}=0 .
\end{gathered}
$$

Here $\nabla^{2}=\partial^{2} / \partial \xi^{2}+\partial^{2} / \partial \eta^{2}$. Equations (9) and (10) are uncoupled. The homogeneous equation (9) constitutes a plane stress problem in elasticity, in which $u_{m}$ and $v_{m}$ are unknown functions. The corresponding homegeneous boundary conditions are

$$
\begin{array}{lll}
u_{m}=0, & \text { or } & \left(\frac{\partial u_{m}}{\partial \xi}+\nu \frac{\partial v_{m}}{\partial \eta}\right) l+\frac{(1-\nu)}{2}\left(\frac{\partial u_{m}}{\partial \eta}+\frac{\partial v_{m}}{\partial \xi}\right) m=0, \\
v_{m}=0, & \text { or } \quad & \frac{(1-\nu)}{2}\left(\frac{\partial u_{m}}{\partial \eta}+\frac{\partial v_{m}}{\partial \xi}\right) l+\left(\nu \frac{\partial u_{m}}{\partial \xi}+\frac{\partial v_{m}}{\partial \eta}\right) m=0 .
\end{array}
$$

In most cases any in-plane rigid body motions of the plate are suppressed, so one has

$$
u_{m}=v_{m}=0 \text {. }
$$

Therefore, only the set of eighth-order partial differential equations (10) has to be solved.

When a plate is in simple harmonic vibration, the forms

$$
w(\xi, \eta, t)=w(\xi, \eta) \mathrm{e}^{\mathrm{i} \omega t}, \quad \psi_{\xi}(\xi, \eta, t)=\psi_{\xi}(\xi, \eta) \mathrm{e}^{\mathrm{i} \omega t}, \quad \psi_{\eta}(\xi, \eta, t)=\psi_{\eta}(\xi, \eta) \mathrm{e}^{\mathrm{i} \omega t},
$$

are introduced. The circular frequency $\omega$ can be expressed in dimensionless form as

$$
\Omega=\omega \sqrt{\rho a^{4} /\left(D_{1}+D_{3}\right)} .
$$

Equations (13) can now be substituted into equations (10). However, as the plate is assumed to vibrate harmonically, the shear modulus $G_{2}$ of the core has to be changed into the complex modulus $G_{2}(1+\mathrm{i} \beta)$; here $\beta$ is the loss factor of the viscoelastic material. The inertia term in equations $(10)\left[\rho a^{4} /\left(D_{1}+D_{3}\right)\right] \partial^{2} w / \partial t^{2}=-\Omega^{2} w$ must also be changed correspondingly into $-\Omega^{2}\left(1+\mathrm{i} \eta^{*}\right) w$; here the quantity $\eta^{*}$ is called the modal loss factor of the plate. The physical significance of the complex frequency $\Omega^{2}\left(1+i \eta^{*}\right)$ has been discussed in reference [10]. The amplitudes $w(\xi, \eta), \psi_{\xi}(\xi, \eta)$ and $\psi_{\eta}(\xi, \eta)$ are all complex quantities. They must satisfy the following equations:

$$
\begin{gathered}
\frac{\partial^{2} \psi_{\xi}}{\partial \xi^{2}}+\frac{(1+\nu)}{2} \frac{\partial^{2} \psi_{\eta}}{\partial \xi \partial \eta}+\frac{(1-\nu)}{2} \frac{\partial^{2} \psi_{\xi}}{\partial \eta^{2}}-g(1+\mathrm{i} \beta)\left(\psi_{\xi}+\frac{\partial w}{\partial \xi}\right)=0 \\
\frac{\partial^{2} \psi_{\eta}}{\partial \eta^{2}}+\frac{(1+\nu)}{2} \frac{\partial^{2} \psi_{\xi}}{\partial \xi \partial \eta}+\frac{(1-\nu)}{2} \frac{\partial^{2} \psi_{\eta}}{\partial \xi^{2}}-g(1+\mathrm{i} \beta)\left(\psi_{\eta}+\frac{\partial w}{\partial \eta}\right)=0 \\
\nabla^{4} w-Y g(1+\mathrm{i} \beta)\left(\frac{\partial \psi_{\xi}}{\partial \xi}+\frac{\partial \psi_{\eta}}{\partial \eta}+\nabla^{2} w\right)-\Omega^{2}\left(1+\mathrm{i} \eta^{*}\right) w=0
\end{gathered}
$$


In the particular case of a symmetrical sandwich plate equations (15) are identical with equations (5-7) of reference [3]. Typical homogeneous boundary conditions of unsymmetrical sandwich plates are as follows: (1) simply supported edges (I),

$$
\begin{gathered}
\partial \psi_{\xi} / \partial \xi\left(l^{2}+\nu m^{2}\right)+\partial \psi_{\eta} / \partial \eta\left(m^{2}+\nu l^{2}\right)+(1-\nu)\left(\partial \psi_{\xi} / \partial \eta+\partial \psi_{\eta} / \partial \xi\right) l m=0 \\
\psi_{s}=0, \quad w=0, \quad \partial^{2} w / \partial n^{2}+\left(\nu / \rho_{s}\right) \partial w / \partial n=0
\end{gathered}
$$

(2) simply supported edges (II),

$$
\begin{gathered}
\partial \psi_{\xi} / \partial \xi\left(l^{2}+\nu m^{2}\right)+\partial \psi_{\eta} / \partial \eta\left(m^{2}+\nu l^{2}\right)+(1-\nu)\left(\partial \psi_{\xi} / \partial \eta+\partial \psi_{\eta} / \partial \xi\right) l m=0 \\
-\left(\partial \psi_{\xi} / \partial \xi-\partial \psi_{\eta} / \partial \eta\right) l m+\frac{1}{2}\left(\partial \psi_{\xi} / \partial \eta+\partial \psi_{\eta} / \partial \xi\right)\left(l^{2}-m^{2}\right)=0 \\
w=0, \quad \partial^{2} w / \partial n^{2}+\left(\nu / \rho_{s}\right) \partial w / \partial n=0
\end{gathered}
$$

(3) clamped edges,

$$
\psi_{n}=0, \quad \psi_{s}=0, \quad w=0, \quad \partial w / \partial n=0
$$

(4) free edges,

$$
\begin{gathered}
\partial \psi_{\xi} / \partial \xi\left(l^{2}+\nu m^{2}\right)+\partial \psi_{\eta} / \partial \eta\left(m^{2}+\nu l^{2}\right)+(1-\nu)\left(\partial \psi_{\xi} / \partial \eta+\partial \psi_{\eta} / \partial \xi\right) l m=0 \\
-\left(\partial \psi_{\xi} / \partial \xi-\partial \psi_{\eta} / \partial \eta\right) l m+\frac{1}{2}\left(\partial \psi_{\xi} / \partial \eta+\partial \psi_{\eta} / \partial \xi\right)\left(l^{2}-m^{2}\right)=0 . \\
-\frac{\partial}{\partial n}\left(\nabla^{2} w\right)-(1-\nu) \frac{\partial}{\partial s}\left[\frac{\partial}{\partial n}\left(\frac{\partial w}{\partial s}\right)\right]+Y g(1+\mathrm{i} \beta)\left[\left(\psi_{\xi}+\frac{\partial w}{\partial \xi}\right) l+\left(\psi_{\eta}+\frac{\partial w}{\partial \eta}\right) m\right]=0 \\
\frac{\partial^{2} w}{\partial n^{2}}+\nu\left(\frac{\partial^{2} w}{\partial s^{2}}+\frac{1}{\rho_{s}} \frac{\partial w}{\partial n}\right)=0
\end{gathered}
$$

(5) at a corner,

$$
w=0, \quad \text { or }\left.\quad\left[\frac{\partial}{\partial n}\left(\frac{\partial w}{\partial s}\right)\right]\right|_{s_{i}+0} ^{s_{i}-0}=0 .
$$

Here $l=\cos (n, \xi)$ and $m=\cos (n, \eta)$ are the direction cosines of the normal $n$ drawn outwards on the boundary of the plate, where $n$ (and $s$ ) are dimensionless. The $\rho_{s}$ is the local radius of curvature of the periphery. Thus to investigate flexural vibration of a viscoelastically damped unsymmetrical sandwich plate, one must first solve equations (15) for a given set of boundary conditions to find the natural frequencies $\Omega$, the modal loss factors $\eta^{*}$, and the corresponding complex modes $w, \psi_{\xi}$ and $\psi_{\eta}$.

\section{THE ASYMPTOTIC SOLUTION OF THE GOVERNING EQUATIONS}

Solving equations (15) is difficult. The exact solution can be obtained only in some particular cases. To obtain an approximate and practical solution and to avoid calculation with complex values, an asymptotic solution with $\mu=i \beta$ as a complex parameter can be introduced. The same procedure has been used in reference [11] to find the loss factors of a sandwich cantilever. One first expands the solution in power series:

$$
\begin{gathered}
w(\xi, \eta)=w_{0}(\xi, \eta)+\mu w_{1}(\xi, \eta)+\mu^{2} w_{2}(\xi, \eta)+\mu^{3} w_{3}(\xi, \eta)+\cdots, \\
\psi_{\xi}(\xi, \eta)=\psi_{\xi 0}(\xi, \eta)+\mu \psi_{\xi 1}(\xi, \eta)+\mu^{2} \psi_{\xi 2}(\xi, \eta)+\mu^{3} \psi_{\xi 3}(\xi, \eta)+\cdots, \\
\psi_{\eta}(\xi, \eta)=\psi_{\eta 0}(\xi, \eta)+\mu \psi_{\eta 1}(\xi, \eta)+\mu^{2} \psi_{\eta 2}(\xi, \eta)+\mu^{3} \psi_{\eta 3}(\xi, \eta)+\cdots, \\
\Omega^{2}=\Omega_{0}^{2}+\mu^{2} \Omega_{2}^{2}+\cdots, \\
\mathrm{i} \eta^{*}=\mu \eta_{1}^{*}+\mu^{3} \eta_{3}^{*}+\cdots .
\end{gathered}
$$


Substituting equations (21) into equations (15) gives the successive equations that the asymptotic solution must satisfy:

$$
\begin{gathered}
L_{3}^{*}\left(w_{0}, \psi_{\xi 0}, \psi_{\eta 0}\right) \equiv \frac{\partial^{2} \psi_{\xi 0}}{\partial \xi^{2}}+\frac{(1+\nu)}{2} \frac{\partial^{2} \psi_{\eta 0}}{\partial \xi \partial \eta}+\frac{(1-\nu)}{2} \frac{\partial^{2} \psi_{\xi 0}}{\partial \eta^{2}}-g\left(\psi_{\xi 0}+\frac{\partial w_{0}}{\partial \xi}\right)=0 \\
L_{4}^{*}\left(w_{0}, \psi_{\xi 0}, \psi_{\eta 0}\right) \equiv \frac{\partial^{2} \psi_{\eta 0}}{\partial \eta^{2}}+\frac{(1+\nu)}{2} \frac{\partial^{2} \psi_{\xi 0}}{\partial \xi \partial \eta}+\frac{(1-\nu)}{2} \frac{\partial^{2} \psi_{\eta 0}}{\partial \xi^{2}}-g\left(\psi_{\eta 0}+\frac{\partial w_{0}}{\partial \eta}\right)=0 \\
L_{5}^{*}\left(w_{0}, \psi_{\xi 0}, \psi_{\eta 0}\right) \equiv \nabla^{4} w_{0}-Y g\left(\frac{\partial \psi_{\xi 0}}{\partial \xi}+\frac{\partial \psi_{\eta 0}}{\partial \eta}+\nabla^{2} w_{0}\right)-\Omega_{0}^{2} w_{0}=0 \\
L_{3}^{*}\left(w_{1}, \psi_{\xi 1}, \psi_{\eta 1}\right)=g\left(\psi_{\xi 0}+\frac{\partial w_{0}}{\partial \xi}\right), \quad L_{4}^{*}\left(w_{1}, \psi_{\xi 1}, \psi_{\eta 1}\right)=g\left(\psi_{\eta 0}+\frac{\partial w_{0}}{\partial \eta}\right) \\
L_{5}^{*}\left(w_{1}, \psi_{\xi 1}, \psi_{\eta 1}\right)=Y g\left(\frac{\partial \psi_{\xi 0}}{\partial \xi}+\frac{\partial \psi_{\eta 0}}{\partial \eta}+\nabla^{2} w_{0}\right)+\Omega_{0}^{2} \eta_{1}^{*} w_{0} \\
L_{3}^{*}\left(w_{2}, \psi_{\xi 2}, \psi_{\eta 2}\right)=g\left(\psi_{\xi 1}+\frac{\partial w_{1}}{\partial \xi}\right), \quad L_{4}^{*}\left(w_{2}, \psi_{\xi 2}, \psi_{\eta 2}\right)=g\left(\psi_{\eta 1}+\frac{\partial w_{1}}{\partial \eta}\right) \\
L_{5}^{*}\left(w_{2}, \psi_{\xi 2}, \psi_{\eta 2}\right)=Y g\left(\frac{\partial \psi_{\xi 1}}{\partial \xi}+\frac{\partial \psi_{\eta 1}}{\partial \eta}+\nabla^{2} w_{1}\right)+\Omega_{0}^{2} \eta_{1}^{*} w_{1}+\Omega_{2}^{2} w_{0} \\
L_{3}^{*}\left(w_{3}, \psi_{\xi 3}, \psi_{\eta 3}\right)=g\left(\psi_{\xi 2}+\frac{\partial w_{2}}{\partial \xi}\right), \quad L_{4}^{*}\left(w_{3}, \psi_{\xi 3}, \psi_{\eta 3}\right)=g\left(\psi_{\eta 2}+\frac{\partial w_{2}}{\partial \eta}\right) \\
L_{5}^{*}\left(w_{3}, \psi_{\xi 3}, \psi_{\eta 3}\right)=Y g\left(\frac{\partial \psi_{\xi 2}}{\partial \xi}+\frac{\partial \psi_{\eta 2}}{\partial \eta}+\nabla^{2} w_{2}\right)+\Omega_{0}^{2}\left(\eta_{1}^{*} w_{2}+\eta_{3}^{*} w_{0}\right)+\Omega_{2}^{2}\left(\eta w_{1}^{*}+w_{1}\right)
\end{gathered}
$$

Here $L_{3}^{*}, L_{4}^{*}$ and $L_{5}^{*}$ are linear partial differential operators defined in equations (22). Substituting equations (21) into the boundary conditions (16)-(20), one finds that, except for the third of equations (19) for free edges which must be expanded in power series of $\mu$, the expressions for all other types of boundary conditions which the successive terms $w_{i}, \psi_{\xi i}, \psi_{\eta i}(i=0,1,2, \ldots)$ must satisfy are the same in form as before. Therefore, from equations (22) and the boundary conditions with respect to $w_{0}, \psi_{\xi 0}, \psi_{\eta 0}$ one can solve first a real eigenvalue problem and obtain all the eigenvalues $\Omega_{0}^{2}$ and corresponding modes $w_{0}, \psi_{\xi 0}$ and $\psi_{\eta 0}$. The modes can be normalized as follows.

If all the boundary conditions of the plate are homogeneous, one can prove that orthogonality exists between two complex modes. This means that if there are two different complex eigenvalues $\Omega_{I}^{2}\left(1+\mathrm{i} \eta_{I}^{*}\right)$ and $\Omega_{I I}^{2}\left(1+\mathrm{i} \eta_{l I}^{*}\right)$ and their corresponding complex modes $w_{I}, \psi_{\xi I}, \psi_{\eta I}$ and $w_{I I}, \psi_{\xi I I}, \psi_{\eta I I}$, then

$$
\iint w_{1} w_{I I} \mathrm{~d} \xi \mathrm{d} \eta=0 .
$$

Equation (26) is analogous to the orthogonality condition in the case of sandwich beams which was given in reference [12]. Therefore, the normalization of the complex modes can be stated as

$$
\iint w^{2} d \xi d \eta=1
$$


Substituting the first of equations (21) into equation (27), one can obtain

$$
\begin{gathered}
\iint w_{0}^{2} \mathrm{~d} \xi \mathrm{d} \eta=1, \quad \iint 2 w_{0} w_{1} \mathrm{~d} \xi \mathrm{d} \eta=0, \\
\iint\left(2 w_{0} w_{2}+w_{1}^{2}\right) \mathrm{d} \xi \mathrm{d} \eta=0, \quad \iint\left(2 w_{0} w_{3}+2 w_{1} w_{2}\right) \mathrm{d} \xi \mathrm{d} \eta=0, \ldots
\end{gathered}
$$

successively. In accordance with equation (28) one can normalize the real mode $w_{0}, \psi_{\xi 0}$ and $\psi_{\eta 0}$. From equations (22),

$$
\iint\left[Y \psi_{\xi 0} L_{3}^{*}\left(w_{0}, \psi_{\xi 0}, \psi_{\eta 0}\right)+Y \psi_{\eta 0} L_{4}^{*}\left(w_{0}, \psi_{\xi 0}, \psi_{\eta 0}\right)-w_{0} L_{5}^{*}\left(w_{0}, \psi_{\xi 0}, \psi_{\eta 0}\right)\right] \mathrm{d} \xi \mathrm{d} \eta=0
$$

Through integrations by parts and other calculations one obtains

$$
\begin{aligned}
\Omega_{0}^{2}= & \iint\left\{\left[\left(\frac{\partial^{2} w_{0}}{\partial \xi^{2}}\right)^{2}+2 \nu \frac{\partial^{2} w_{0}}{\partial \xi^{2}} \frac{\partial^{2} w_{0}}{\partial \eta^{2}}+\left(\frac{\partial^{2} w_{0}}{\partial \eta^{2}}\right)^{2}+2(1-\nu)\left(\frac{\partial^{2} w_{0}}{\partial \xi \partial \eta}\right)^{2}\right]\right. \\
& +Y\left[\left(\frac{\partial \psi_{\xi 0}}{\partial \xi}\right)^{2}+2 \nu \frac{\partial \psi_{\xi 0}}{\partial \xi} \frac{\partial \psi_{\eta 0}}{\partial \eta}+\left(\frac{\partial \psi_{\eta 0}}{\partial \eta}\right)^{2}+\frac{(1-\nu)}{2}\left(\frac{\partial \psi_{\xi 0}}{\partial \eta}+\frac{\partial \psi_{\eta 0}}{\partial \xi}\right)^{2}\right] \\
& \left.+Y g\left[\left(\psi_{\xi 0}+\frac{\partial w_{0}}{\partial \xi}\right)^{2}+\left(\psi_{\eta 0}+\frac{\partial w_{0}}{\partial \eta}\right)^{2}\right]\right\} \mathrm{d} \xi \mathrm{d} \eta .
\end{aligned}
$$

The sum of the first two integrals in equation (33) is proportional to the strain energy in the faces 1 and 3 due to the normalized real mode $w_{0}, \psi_{\xi 0}$ and $\psi_{\eta 0}$, while the third integral is proportional to the strain energy in the core. Both proportionality coefficients are $2 /\left(D_{1}+D_{3}\right)$. This is obvious from comparison of equations (33) and (7).

Next one can solve equations (23). First the value of $\eta_{1}^{*}$ must be determined. By using a similar procedure as before, when equation (32) was obtained, one gets a relation from equation (23):

$$
\begin{gathered}
\iint\left[Y \psi_{\xi 0} L_{3}^{*}\left(w_{1}, \psi_{\xi 1}, \psi_{\eta 1}\right)+Y \psi_{\eta 0} L_{4}^{*}\left(w_{1}, \psi_{\xi 1}, \psi_{\eta 1}\right)-w_{0} L_{5}^{*}\left(w_{1}, \psi_{\xi 1}, \psi_{\eta 1}\right)\right] \mathrm{d} \xi \mathrm{d} \eta \\
=\iint\left\{\boldsymbol{Y g}\left[\psi_{\xi 0}\left(\psi_{\xi 0}+\frac{\partial w_{0}}{\partial \xi}\right)+\psi_{\eta 0}\left(\psi_{\eta 0}+\frac{\partial w_{0}}{\partial \eta}\right)-w_{0}\left(\frac{\partial \psi_{\xi 0}}{\partial \xi}+\frac{\partial \psi_{\eta 0}}{\partial \eta}+\nabla^{2} w_{0}\right)\right]\right. \\
\left.-\Omega_{0}^{2} \eta_{1}^{*} w_{0}^{2}\right\} \mathrm{d} \xi \mathrm{d} \eta .
\end{gathered}
$$

From equation (34) one obtains

$$
\eta_{1}^{*}=\frac{1}{\Omega_{0}^{2}} \iint Y g\left[\left(\psi_{\xi 0}+\frac{\partial w_{0}}{\partial \xi}\right)^{2}+\left(\psi_{\eta 0}+\frac{\partial w_{0}}{\partial \eta}\right)^{2}\right] \mathrm{d} \xi \mathrm{d} \eta,
$$

$\eta_{1}^{*}$ is evidently a fraction of elastic strain energy attributable to the viscoelastic core when the damped plate deforms in the mode $w_{0}, \psi_{\xi 0}$ and $\psi_{\eta 0}$. Up to now all the first terms of each expression in equations (21) have been obtained. If less accuracy is acceptable, the approximate solution can be expressed as

$$
\begin{aligned}
& w(\xi, \eta)=w_{0}(\xi, \eta), \quad \psi_{\xi}(\xi, \eta)=\psi_{\xi 0}(\xi, \eta), \quad \psi_{\eta}(\xi, \eta)=\psi_{\eta 0}(\xi, \eta), \\
& \Omega^{2}=\Omega_{0}^{2}, \quad \eta^{*}=\eta_{1}^{*} \beta \text {. }
\end{aligned}
$$

In references [7] and [8] the Modal Strain Energy (MSE) Method is suggested for analysis of viscoelastically damped structures. For viscoelastically damped sandwich plates, adopting the simplified scheme of this paper, one obtains equations (33), (35) and (36), which are the same as the results obtained by means of MSE. Therefore, MSE can be considered to be the first-order approximation of the asymptotic solution presented in this paper. 
To improve accuracy, one must calculate successive terms of the asymptotic solution. Having determined the value of $\eta_{1}^{*}$, one can obtain the unique solution for $w_{1}, \psi_{\xi 1}$ and $\psi_{\eta 1}$ of all modes in accordance with equations (23) and corresponding boundary conditions. The general solution for $w_{1}, \psi_{\xi 1}$ and $\psi_{\eta 1}$ may be written in the following form:

$$
\begin{gathered}
w_{1}(\xi, \eta)=w_{1 s}(\xi, \eta)+C_{1} w_{0}(\xi, \eta), \quad \psi_{\xi 1}(\xi, \eta)=\psi_{\xi 1 s}(\xi, \eta)+C_{1} \psi_{\xi 0}(\xi, \eta) \\
\psi_{\eta 1}(\xi, \eta)=\psi_{\eta 1 s}(\xi, \eta)+C_{1} \psi_{\eta 0}(\xi, \eta) .
\end{gathered}
$$

Here $w_{1 s}, \psi_{\xi 1 s}$ and $\psi_{\eta 1 s}$ are a set of particular solutions, where $C_{1}$ is an undetermined constant. The value of $C_{1}$ can be given by means of the normalization condition, equation (29). Then the expressions for $w_{1}, \psi_{\xi 1}$ and $\psi_{\eta 1}$ can be determined completely.

Thereafter one solves equation (24). Through calculations similar to those previously, the expression for $\Omega_{2}^{2}$ is first obtained.

$$
\Omega_{2}^{2}=Y g \iint\left[\left(\psi_{\xi 0}+\frac{\partial w_{0}}{\partial \xi}\right)\left(\psi_{\xi 1}+\frac{\partial w_{1}}{\partial \xi}\right)+\left(\psi_{\eta 0}+\frac{\partial w_{0}}{\partial \eta}\right)\left(\psi_{\eta 1}+\frac{\partial w_{1}}{\partial \eta}\right)\right] \mathrm{d} \xi \mathrm{d} \eta .
$$

Using the normalization condition (30), one can obtain the unique solution for $w_{2}, \psi_{\xi 2}$ and $\psi_{\eta 2}$ in accordance with equations (24) and corresponding boundary conditions.

Before solving equations (25) one determines the value of $\eta_{3}^{*}$;

$$
\begin{aligned}
\eta_{3}^{*}= & -\eta_{1}^{*}\left(\iint w_{0} w_{2} \mathrm{~d} \xi \mathrm{d} \eta+\frac{\Omega_{2}^{2}}{\Omega_{0}^{2}}\right) \\
& +\frac{Y g}{\Omega_{0}^{2}} \iint\left[\left(\psi_{\xi 0}+\frac{\partial w_{0}}{\partial \xi}\right)\left(\psi_{\xi 2}+\frac{\partial w_{2}}{\partial \xi}\right)+\left(\psi_{\eta 0}+\frac{\partial w_{0}}{\partial \eta}\right)\left(\psi_{\eta 2}+\frac{\partial w_{2}}{\partial \eta}\right)\right] \mathrm{d} \xi \mathrm{d} \eta .
\end{aligned}
$$

Then, using equation (31), one can also obtain the unique solution for $w_{3}, \psi_{53}$ and $\psi_{\eta 3}$. If the calculation is not to be continued, one is left with the expressions for the asymptotic solution given explicitly in equations $(21)$, without the residual terms indicated by $(\cdots)$ on their right sides. The last of equations (21) may be rewritten as

$$
\eta^{*}=\eta_{1}^{*} \beta /\left[1+\left(\eta_{3}^{*} / \eta_{1}^{*}\right) \beta^{2}\right]
$$

By calculating in accordance with equation (40), one can often obtain better results.

\section{AN EXAMPLE}

Consider a rectangular viscoelastically damped sandwich plate with simple support (I) along all edges. Its length and width are $a$ and $b$ respectively. Define $\theta=b / a$. Introduce a dimensionless co-ordinate system $0 \xi \eta$ and let the edges of the plate to be $\xi=0,1$ and $\eta=0, \theta$. One can obtain the asymptotic solution for the natural frequencies, modes and modal loss factors of this plate as follows:

$$
\begin{gathered}
\Omega_{0}^{2}=\alpha_{p q}^{2}\left(1+\frac{Y g}{\alpha_{p q}+g}\right), \quad w_{0}(\xi, \eta)=\frac{2}{\sqrt{\theta}} \sin p \pi \xi \sin \frac{q \pi \eta}{\theta} \\
\psi_{\xi 0}(\xi, \eta)=-\frac{2 p \pi g}{\sqrt{\theta}\left(\alpha_{p q}+g\right)} \cos p \pi \xi \sin \frac{q \pi \eta}{\theta} \\
\psi_{\eta v}(\xi, \eta)=-\frac{2 q \pi g}{\theta \sqrt{\theta}\left(\alpha_{p q}+g\right)} \sin p \pi \xi \cos \frac{q \pi \eta}{\theta}
\end{gathered}
$$




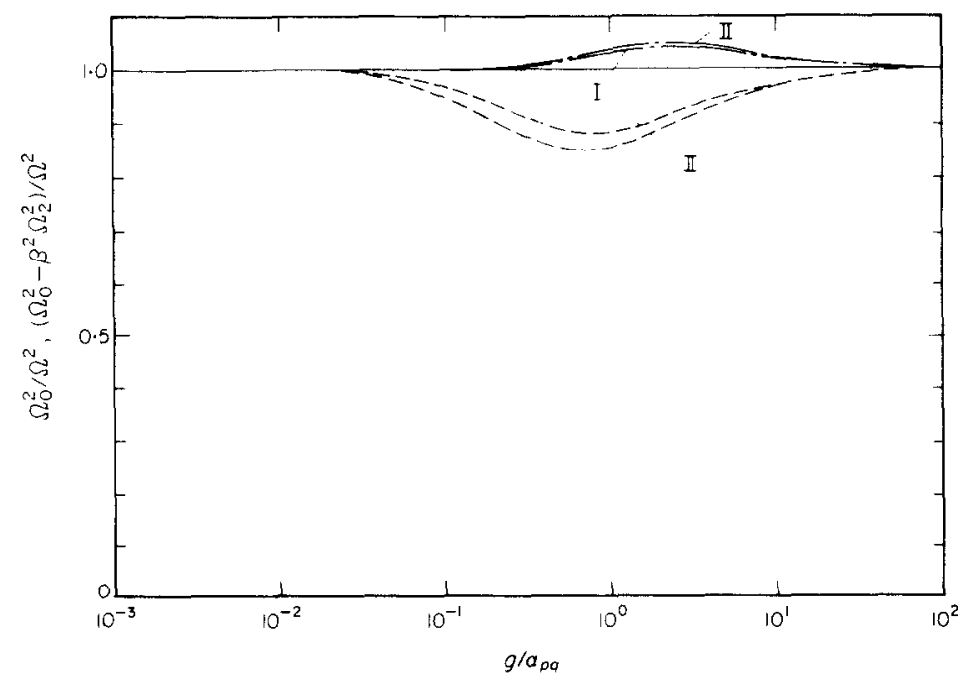

Figure 2. Ratio of the asymptotic solutions of natural frequencies to the exact ones $v s$. the variable $g / \alpha_{p q}$. $-\cdots, \Omega_{0}^{2} / \Omega^{2} ;-\cdot,\left(\Omega_{0}^{2}-\beta^{2} \Omega_{2}^{2}\right) / \Omega^{2} .1, Y=3 \cdot 5 ; \mathrm{II}, Y=10(\beta=1 \cdot 0)$.

here $\alpha_{p q}=p^{2} \pi^{2}+q^{2} \pi^{2} / \theta^{2}$, and $p$ and $q$ are positive integers;

$$
\begin{gathered}
\eta_{1}^{*}=\alpha_{p q} Y g /\left[\left(\alpha_{p q}+g\right)^{2}+Y g\left(\alpha_{p q}+g\right)\right], \quad w_{1}(\xi, \eta)=0 . \\
\psi_{\xi 1}(\xi, \eta)=-\frac{2 p \pi g \alpha_{p q}}{\sqrt{\theta}\left(\alpha_{p q}+g\right)^{2}} \cos p \pi \xi \sin \frac{q \pi \eta}{\theta}, \\
\psi_{\eta 1}(\xi, \eta)=-\frac{2 q \pi g \alpha_{p q}}{\theta \sqrt{\theta}\left(\alpha_{p q}+g\right)^{2}} \sin p \pi \xi \cos \frac{q \pi \eta}{\theta} ; \\
\Omega_{2}^{2}=-Y g^{2} \alpha_{p q}^{3} /\left(\alpha_{p q}+g\right)^{3}, \quad w_{2}(\xi, \eta)=0, \\
\psi_{\xi 2}(\xi, \eta)=\frac{2 p \pi g^{2} \alpha_{p q}}{\sqrt{\theta}\left(\alpha_{p q}+g\right)^{3}} \cos p \pi \xi \sin q \pi \eta / \theta, \\
\psi_{\eta 2}(\xi, \eta)=\frac{2 q \pi g^{2} \alpha_{p q}}{\theta \sqrt{\theta}\left(\alpha_{p q}+g\right)^{3}} \sin p \pi \xi \cos \frac{q \pi \eta}{\theta} ; \\
\eta_{3}^{*}=\eta_{1}^{*}(1+Y) g^{2} /\left[\left(\alpha_{p q}+g\right)^{2}+Y g\left(\alpha_{p q}+g\right)\right], \\
\psi_{\xi 3}(\xi, \eta)=-\frac{2 p \pi g^{3} \alpha_{p q}}{\sqrt{\theta}\left(\alpha_{p q}+g\right)^{4}} \cos p \pi \xi \sin \frac{q \pi \eta}{\theta}, \\
\psi_{\eta 3}(\xi, \eta)=-\frac{2 q \pi g^{3} \alpha_{p q}}{\theta \sqrt{\theta}\left(\alpha_{p q}+g\right)^{4}} \sin p \pi \xi \cos \frac{q \pi \eta}{\theta}, \ldots
\end{gathered}
$$

As this problem is simple, the exact solution of it can be obtained directly from equations (15)

$$
\begin{gathered}
\Omega^{2}=\alpha_{p q}^{2}\left\{1+\frac{Y g\left[\alpha_{p q}+g\left(1+\beta^{2}\right)\right]}{\left(\alpha_{p q}+g\right)^{2}+g^{2} \beta^{2}}\right\}, \quad w(\xi, \eta)=\frac{2}{\sqrt{\theta}} \sin p \pi \xi \sin \frac{q \pi \eta}{\theta}, \\
\psi_{\xi}(\xi, \eta)=-\frac{2 p \pi g(1+\mathrm{i} \beta)}{\sqrt{\theta}\left[\alpha_{p q}+g(1+\mathrm{i} \beta)\right]} \cos p \pi \xi \sin \frac{q \pi \eta}{\theta} \\
\psi_{\eta}(\xi, \eta)=-\frac{2 q \pi g(1+\mathrm{i} \beta)}{\theta \sqrt{\theta}\left[\alpha_{p q}+g(1+\mathrm{i} \beta)\right]} \sin p \pi \xi \cos \frac{q \pi \eta}{\theta} \\
\eta^{*}=\alpha_{p q} Y g \beta /\left[\left(\alpha_{p q}+g\right)^{2}+Y g\left(\alpha_{p q}+g\right)+(1+Y) g^{2} \beta^{2}\right] .
\end{gathered}
$$




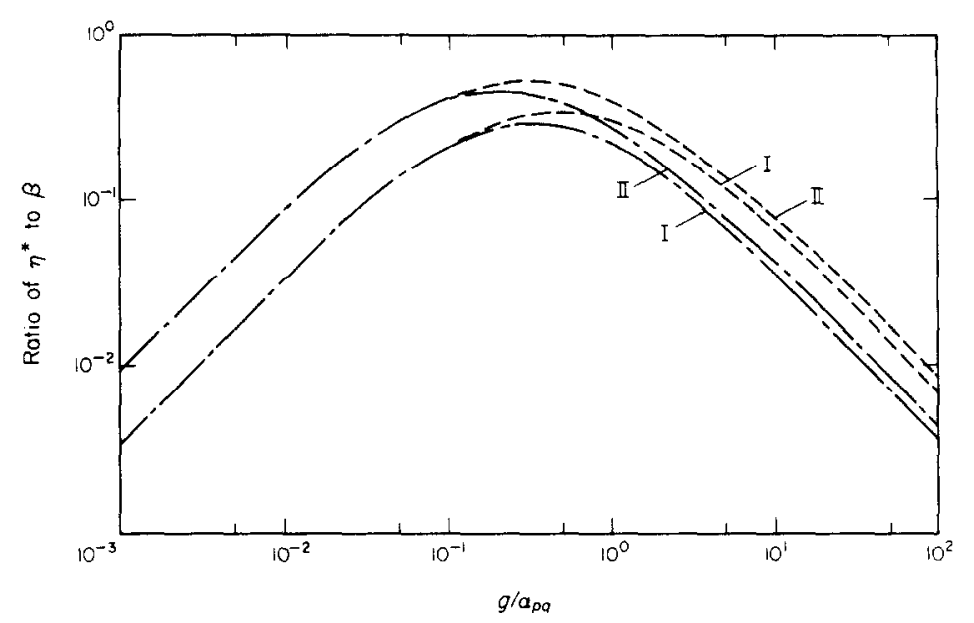

Figure 3. Ratio of $\eta^{*}$ to $\beta$ vs. the variable $g / \alpha_{p q} \cdot \beta=1 \cdot 0 .-\cdots, \eta_{1}^{*} ;-\cdot-, \eta_{1}^{*} /\left[1+\left(\eta_{3}^{*} / \eta_{1}^{*}\right) \beta^{2}\right] . \mathrm{I}, Y=3 \cdot 5$; II, $Y=10$.

To examine the accuracy of the asymptotic solution obtained, numerical calculation was done for two plates, both with $\beta=1 \cdot 0$, but with $Y=3.5$ or 10 respectively. In Figure 2 the curves representing the variation of $\Omega_{0}^{2} / \Omega^{2}$ and $\left(\Omega_{0}^{2}-\beta^{2} \Omega_{2}^{2}\right) / \Omega^{2}$ with the variable $g / \alpha_{p q}$ are given; here $\Omega^{2}$ is the exact solution as shown in equations (45). Obviously the values $\Omega_{0}^{2}-\beta^{2} \Omega_{2}^{2}$ approximate to the exact values $\Omega^{2}$ better than the values $\Omega_{0}^{2}$, which are equivalent to the result obtained by the MSE Method. Figure 3 shows $\eta^{*} / \beta$ as a function of the variable $g / \alpha_{p q}$, where the values of $\eta^{*}$ are calculated according to $\eta_{1}^{*} \beta$, or equation (40). The latter in this example is just the exact expression of $\eta^{*}$ as shown in the last of equations (45). Figure 2 and Figure 3 are applicable to all modes of simply supported rectangular plates with various values of $\theta$.

\section{CONCLUSIONS}

In this paper a set of simplified governing equations and corresponding boundary conditions of viscoelastically damped unsymmetrical sandwich plates in flexural vibration are given. To avoid calculation with complex values, an asymptotic solution of the simplified governing equations has been introduced, with the loss factor of the viscoelastic material of the core used as a parameter. In simpler cases the asymptotic solution in analytical form can be obtained. In other cases one can use approximate methods to obtain numerical solutions. If in the asymptotic solution only the first terms of all quantities are adopted, then the result is identical in principle with that as given in accordance with the Modal Strain Energy (MSE) Method. However, when the value of the loss factor $\beta$ of the viscoelastic material is larger, then the errors in the values of the natural frequencies and modal loss factors are somewhat appreciable in a certain range of the value of $g$. By taking more terms of the asymptotic solution, with successive calculations, accuracy can be improved. With the character of the free flexural vibrations of a viscoelastically damped sandwich plate thus established, one can further analyze the response of the plate to various types of dynamic loads to provide a reliable basis for design.

\section{REFERENCES}

1. D. J. MEAD 1982 Vibration Control (I), in Noise and Vibration (R. G. White and J. G. Walker, editors). Chichester: Ellis Horwood. See chapter 25, pp. 673-683. 
2. P. J. ToRviK (editor) 1980 Damping Applications for Vibration Control. New York: ASME United Engineering Center.

3. D. J. MEAD 1972 Journal of Sound and Vibration 24, 275-295. The damping properties of elastically supported sandwich plates.

4. Y. V. K. S. RAO and B. C. NAKRA 1973 Archives of Mechanics 25(2), 213-225. Theory of vibratory bending of unsymmetrical sandwich plates.

5. Y. V. K. S. RAO and B. C. NAKRA 1974 Journal of Sound and Vibration 34, 309-326. Vibrations of unsymmetrical sandwich beams and plates with viscoelastic cores.

6. E. IOANNIDES and P. GRoOTEnhuis 1979 Journal of Sound and Vibration 67, 203-218. A finite element analysis of the harmonic response of damped three-layer plates.

7. C. D. JOHNSON and D. A. KIENIIOLZ 1982 American Institute of Aeronautics \& Astronautics Journal 20(9), 1284-1290. Finite element prediction of damping in structures with constrained viscoelastic layers.

8. D. A. Kienholz, C. D. Johnson and J. C. PAREkH 1983 Proceeding of 24th AIAA/ASME / ASCE / AHS Structures, Structural Dynamics and Materials Conference 83-0904. Design methods for viscoelastically damped plates.

9. S. MARKUS and T. NANASI 1981 Journal of Sound and Vibration 76, 421-441. Significance of in-plane inertia forces in the vibration analysis of three-layered circular plates.

10. D. J. MEAD and S. MARKUS 1969 Journal of Sound and Vibration 10, 163-175. The forced vibration of a three-layer, damped sandwich beam with arbitrary boundary conditions.

11. V. ORAVSK Y, S. MARKUS and O. Simkova 1974 Journal of Sound and Vibration 33, 335-352. A new approximate method of finding the loss factors of a sandwich cantilever.

12. D. J. MEAD 1980 Notes of the Seminar on Vibration Control, Applied Research Laboratory, Pennsylvania State University. Vibration control with sandwich beams and plates. 